\title{
Minor effect of inaccurate fixation on VEP-based acuity estimates
}

\author{
Amal A. Elgohary $\cdot$ Sven P. Heinrich $\mathbb{D}$
}

Received: 15 May 2020/Accepted: 25 September 2020/Published online: 10 October 2020

(C) The Author(s) 2020

\begin{abstract}
Purpose VEP-based estimation of visual acuity may be used in cases of suspected malingering to objectify subjective complaints. In such an application, a lack of cooperation needs to be expected. The same may apply to young children with suspected functional impairments. In the present study, we assessed how inaccurate fixation affects the acuity estimates obtained with a VEP technique.

Methods VEP-based acuity estimates were obtained by stimulating with a series of different check sizes using a 'stepwise sweep' protocol. Sixteen participants were tested with normal and degraded vision under five different fixation conditions (central fixation and eccentric fixation at top, bottom, right, and left edge of the stimulus area).

Results The majority of individual acuity estimates with eccentric fixation differed by less than 0.1 $\log$ MAR from central fixation, and almost all
\end{abstract}

\footnotetext{
A. A. Elgohary

Department of Vision Science, Research Institute of Ophthalmology, Giza, Cairo, Egypt
}

S. P. Heinrich ( $ه)$

Eye Center, Medical Center, University of Freiburg, Killianstr. 5, 79106 Freiburg, Germany

e-mail: sven.heinrich@uni-freiburg.de

S. P. Heinrich

Faculty of Medicine, University of Freiburg, Freiburg,

Germany estimates differed by less than $0.3 \log$ MAR. Median estimates with eccentric fixation differed only slightly (up to $0.08 \log$ MAR) and, except for top fixation with normal vision, non-significantly. However, data quality was lower with eccentric fixation, which increased the probability that no acuity estimate could be derived from the recording.

Conclusion VEP-based acuity estimates are relatively insensitive to eccentric fixation. Unnoticed deviations from central fixation in routine applications will probably be smaller than in the present study and will have even less impact on the outcome.

Keywords Objective acuity testing - Visual evoked potentials - Eccentric fixation - Visual acuity · malingering $\cdot$ functional impairment

\section{Introduction}

VEP-based estimation of visual acuity is increasingly popular as a technique in cases of suspected malingering or when a patient is unable to cooperate during standard psychophysical acuity testing [1]. Within certain limits, the reliability of the approach has been demonstrated in a number of studies (e.g., [2-4]), except with disorders that are associated with distortions of the visual percept, such as amblyopia [5, 6] 
and probably also when the reason for the impairment has a locus beyond the primary visual cortex.

During VEP measurements, patients are normally asked to fixate the center of the screen, where a fixation mark is usually provided. Given that suspected malingering is one of the prime scenarios for the use of VEP-based acuity estimation, it is an important question whether incorrect fixation leads to a sizable effect on the outcome of the test. In addition to typical cases of malingering as encountered in the normal clinical routine, misrepresentation of visual impairments by athletes in the classification of participants in vision impaired sports [7] is another potential field of application of VEP-based techniques. Furthermore, incorrect fixation may also occur in young children and patients with mental disabilities or certain oculomotor disorders.

While the problem may in principle be ameliorated by increasing the total stimulus size, this is often not feasible due to technical limitations. For instance, trying to achieve this by moving the screen closer to the patient will increase the angular size of the pixels and thus limit the range of visual resolutions that can be tested.

The present study aimed at addressing this question by obtaining VEP-based acuity estimates from healthy cooperative participants with normal and artificially reduced acuity who are instructed to fixate eccentrically.

\section{Methods}

\section{Participants}

Sixteen normal participants (age range 23-38 years) with normal or corrected-to-normal visual acuity $(\log$ MAR $\leq 0.0$ as measured with the Freiburg Acuity and Contrast Test (FrACT) [8]) participated in the study. All reported free of ophthalmological or neurological disorders and provided written informed consent. The study had been approved by the local institutional review board at the University of Freiburg and followed the tenets of the Declaration of Helsinki. The eye with better visual acuity, or the right eye when both eyes had equal acuity, was selected as study eye.
Stimuli and procedure

VEP-based estimation of visual acuity is not covered by the standard ISCEV VEP standard [9], and an ISCEV extended protocol [10] was only published very recently. We used the procedure described in detail by Bach et al. [11], which is within the specifications of the ISCEV extended protocol. In short, checkerboard onset stimuli were displayed monocularly at a frequency of $7.5 \mathrm{~Hz}$ on a CRT monitor with a Michelson contrast of $40 \%$ and a frame rate of $75 \mathrm{~Hz}$. The size of the stimulus area was $19^{\circ} \times 15^{\circ}$. Six logarithmically approximately equidistant check sizes of $0.046^{\circ}$ to $0.37^{\circ}$ were used. The observation distance was $114 \mathrm{~cm}$. Participants were supplied with a near addition to adjust the refraction to the monitor distance. They were tested with normal vision and with vision artificially blurred using Bangerter occluders (grade 0.4). Five different fixation conditions, namely central, right, left, top and bottom, were tested. The order of the 10 different conditions (5 fixation locations $\times 2$ acuity levels) was randomized individually for each participant. The VEPs were recorded with a Laplacian electrode montage $(\mathrm{Oz}$ versus $(\mathrm{O} 1+\mathrm{O} 2) / 2)$.

Eccentric fixation was achieved by asking the participant to fixate a target that was attached to the edge of the stimulus area on the respective side, so that the horizontal or vertical meridian of the visual field aligned with the edge of the stimulus area. For instance, for left eccentric fixation, the fixation target was in the middle of the left edge of the stimulus area, such that the vertical meridian of the visual field coincides with the edge of the stimulus area and stimulation was limited to the right hemifield.

VEP analysis and acuity estimation

Following Bach et al. [11], all artifact-free 1-s intervals of the steady-state response to a given check size were averaged, and Fourier analysis was applied to extract the response at the stimulation frequency $(7.5 \mathrm{~Hz})$. A noise correction was applied to the amplitudes [12], and response significance was determined [13]. Because in the degraded vision condition most check sizes were too small to be resolved, the chance of spurious significances increased (multiple testing problem). In a few cases, we therefore had to manually correct for this by treating the respective 
data points as not having a statistically significant response.

The resulting tuning curve (amplitude vs. spatial frequency) was extrapolated to zero amplitude using Bach et al.'s [11] heuristic algorithm. The corresponding spatial frequency $\mathrm{SF}_{0}$ was then converted into an acuity estimate using an empirically determined conversion factor that had been determined by that same study using the relationship $\log \mathrm{MAR}=$ $\log \left(17.6 / \mathrm{SF}_{0}\right)[11]$.

\section{Statistical assessment}

The present study assessed a diagnostic procedure that is usually applied to individual persons. Consequently, the question of statistical significance on the group level was not the primary concern. For completeness, however, we performed pairwise comparisons between the acuity estimates obtained with eccentric fixation and the corresponding acuity estimate obtained with central fixation to identify those eccentric fixation locations where the pattern of effects is consistent enough to result in a significant effect on the group level. This was done by applying permutation tests that do not rely on the assumption of a normal distribution [14]. Confidence intervals for differences in acuity estimates were obtained through bootstrapping [14].

\section{Results}

As shown in Fig. 1 for a sample participant, VEP responses with eccentric fixation were generally smaller than with central fixation, albeit with exceptions in particular with top fixation. With normal vision, in two out of 80 recordings (16 participants $\times 5$ fixation locations), no acuity estimate could be obtained. With degraded vision, the respective number was 14 . Generally, visual inspection suggests that data quality was lower with eccentric fixation (see example in Fig. 2).

We compared the participants' standard acuity under normal vision conditions as measured with the FrACT to the respective VEP-based acuity estimates obtained with central fixation. In the majority of participants, both agreed within the limits reported by Bach et al. [11]. For six participants (5 of them with a $\log$ MAR value below -0.3 , i.e., very good acuity substantially beyond the range covered by the VEP stimuli), acuity with normal vision was underestimated by the VEP method.

Figure 3 provides an overview of $\log$ MAR differences between VEP-based estimates of eccentric and central fixation for all individual participants. With normal vision, the absolute $\log$ MAR difference was below 0.1 in $75 \%$ of the successful acuity estimates, with the remainder below 0.3 . With degraded vision, the absolute logMAR difference was below 0.1 in $60 \%$ of the successful acuity estimates and below 0.3 in another $31 \%$.

On the group level, in normal vision, the median $\log$ MAR differences with right, left, top and bottom eccentric fixation were 0.02 (95\% CI, $-0.02 \ldots 0.06$; $p=0.09), \quad 0.05 \quad(0.01 \ldots 0.09 ; \quad p=0.02), \quad 0.08$ $(0.04 \ldots 0.11 ; p<0.001)$ and $0.03(-0.01 \ldots 0.06$; $p=0.53)$, respectively. In degraded vision, the median $\operatorname{logMAR}$ differences with right, left, top and bottom eccentric fixations were $0.00 \quad(95 \%$ CI,$-0.06 \ldots 0.12 ; \quad p=0.41), \quad 0.04 \quad(0.00 \ldots 0.21$; $p=0.01), 0.00(-0.02 \ldots 0.15 ; p=0.12)$ and 0.00 $(-0.05 \ldots 0.11 ; p=0.70)$, respectively. Only the effect with top fixation and normal vision was significant with a Bonferroni correction.

\section{Discussion}

In this study, the employed four eccentric fixations were chosen such that stimulation was limited to one half of the visual field in each condition. Stimuli extended from the respective meridian to moderate eccentricities as defined by the size of the stimulus field.

The median increase in $\log$ MAR estimates was in the range of $0.02-0.08$ for normal vision and 0.00-0.04 for degraded, which (in the absence of knowledge about incorrect fixation) would for most eccentric fixation conditions imply a slightly worse acuity, albeit only statistically significant for top fixation with normal vision. The magnitude of the median effect was less than one line on an acuity chart and thus not very relevant in most cases in which VEP-based acuity estimated would be obtained routinely in individual patients. For comparison, the International Council of Ophthalmology proposes that a mean difference of 0.05 should be taken as the limit for accepting two acuity tests as being equivalent [16]. 


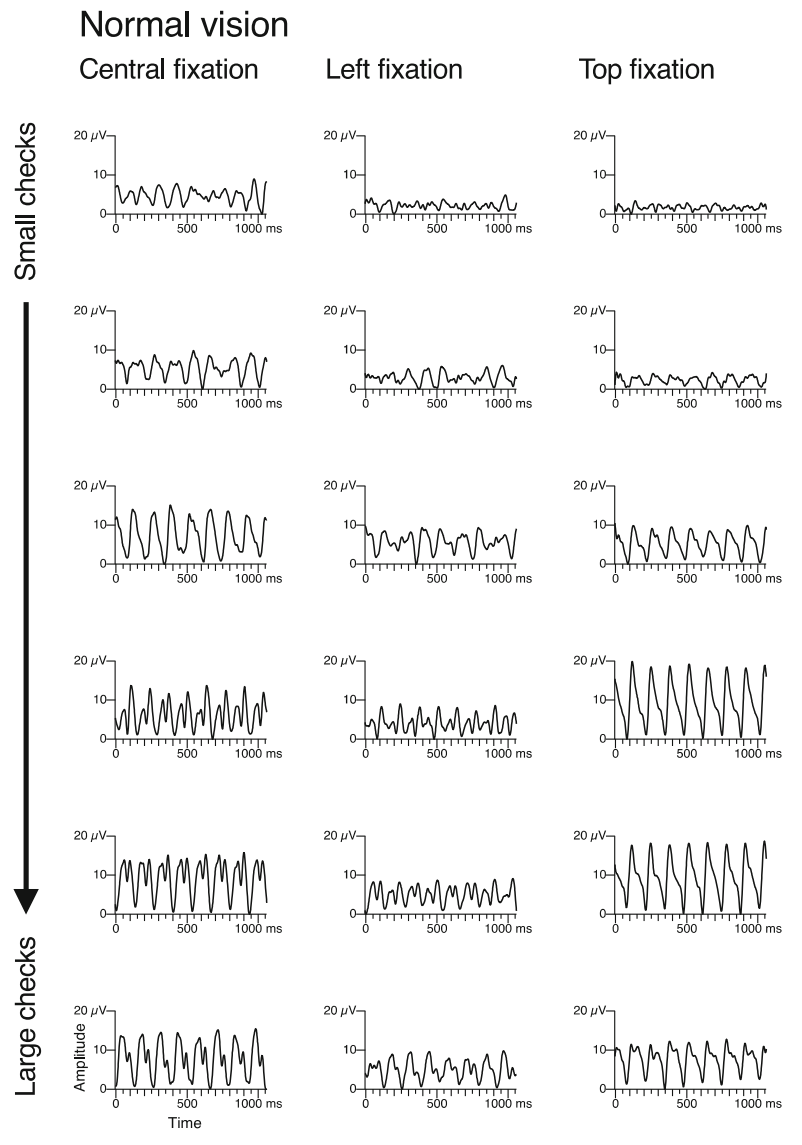

Fig. 1 Sample traces of one participant, obtained with central, left and top fixation. Check size increases from top to bottom. For large checks with normal vision, responses are clearly recognizable. With smaller checks or with degraded vision, responses are reduced or absent. What looks like a response to the smallest checks in some traces is in fact not a $7.5-\mathrm{Hz}$ stimulus response, but an unrelated oscillation at a higher

The situation might be different in group comparisons, as opposed to the assessment of individual patients, if the fixation behavior between groups differs, potentially resulting in a misleading finding of a statistically significant (albeit small) acuity difference.

The median increase in logMAR estimates does not represent the full picture, though. As the examples in Fig. 3 illustrate, there is a considerable interindividual variability. However, in the majority of individual acuity estimates, the absolute logMAR difference was below 0.1 , and in almost all cases it was below 0.3 . The latter value is the approximate range within which VEP-based estimates match psychophysical acuity in $95 \%$ of the cases [11].

\section{Degraded vision}
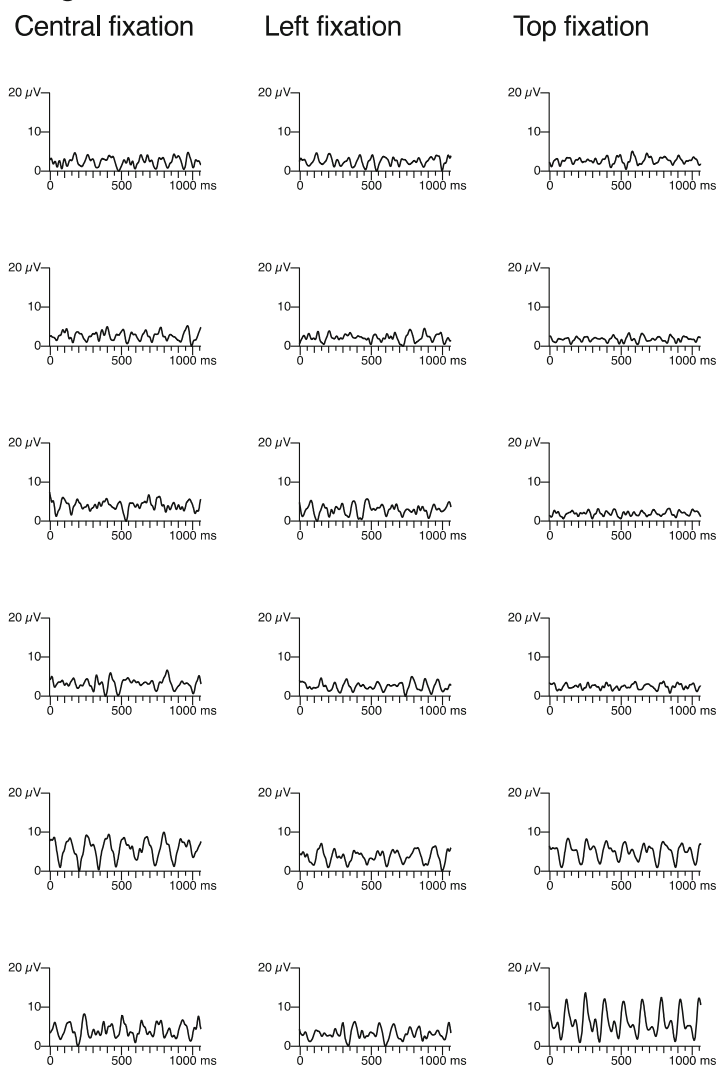

frequency, most likely alpha activity [15]. As an interesting side detail (unrelated to the topic of the present study), a superposition of the presumed alpha activity and the stimulus response probably underlies the beating waves in some traces. Tuning curves of the same participant are shown in the top set of graphs in Fig. 2

Importantly, the effects may have either direction (higher or lower values than with central fixation). In our data set, this is particularly obvious with bottom fixation. A large contribution to these effects is likely to come from general measurement variability rather than from eccentric fixation. The lower data quality with eccentric fixation probably also increases the risk of occasional 'outliers' and explains why no acuity estimate could be obtained in a number of recordings with eccentric fixation.

The use of a Laplacian montage probably contributed beneficially to the study outcome as it is known to yield a better signal-to-noise ratio than a recording from $\mathrm{Oz}$ only (referenced, for instance, to a frontal electrode) [17]. Especially with right or left 

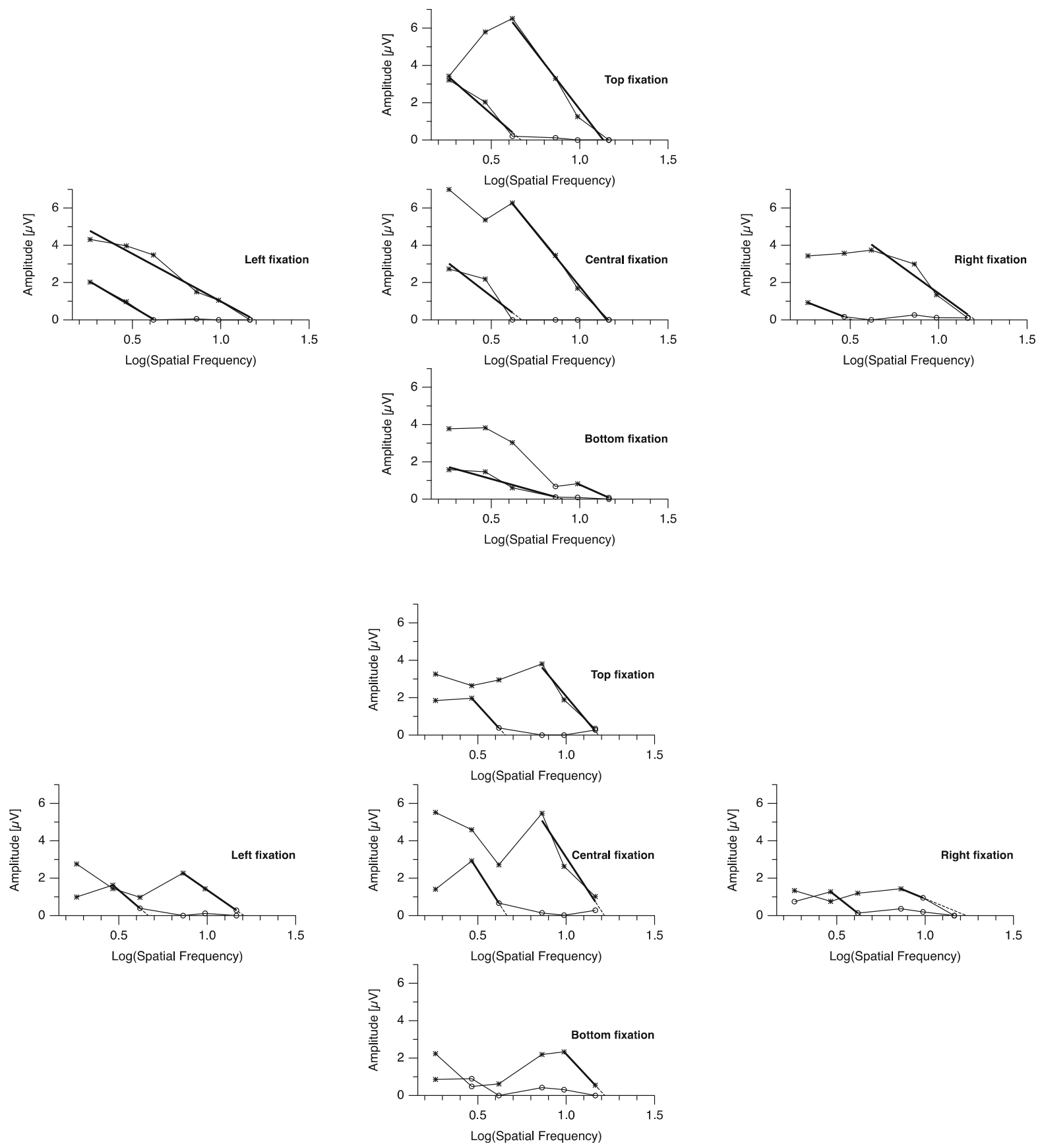

Fig. 2 Comparison of tuning curves. Each group of five graphs shows data of all five fixation conditions of one sample participant. Within each graph, the top curve was obtained with normal vision, and the lower curve with degraded vision. Asterisk markers indicate statistically significant responses. Bold lines represent straight lines fitted to the descending slope of the tuning curve. Dashed lines show the extrapolation to the abscissa, with the intercept yielding the threshold estimate. With normal vision, eccentric fixation typically reduces the amplitudes while leaving the threshold largely unchanged. This is similar in degraded vision, although variability is higher and in some cases the algorithm may fail to yield and acuity estimate (lower set of graphs, bottom graph) 

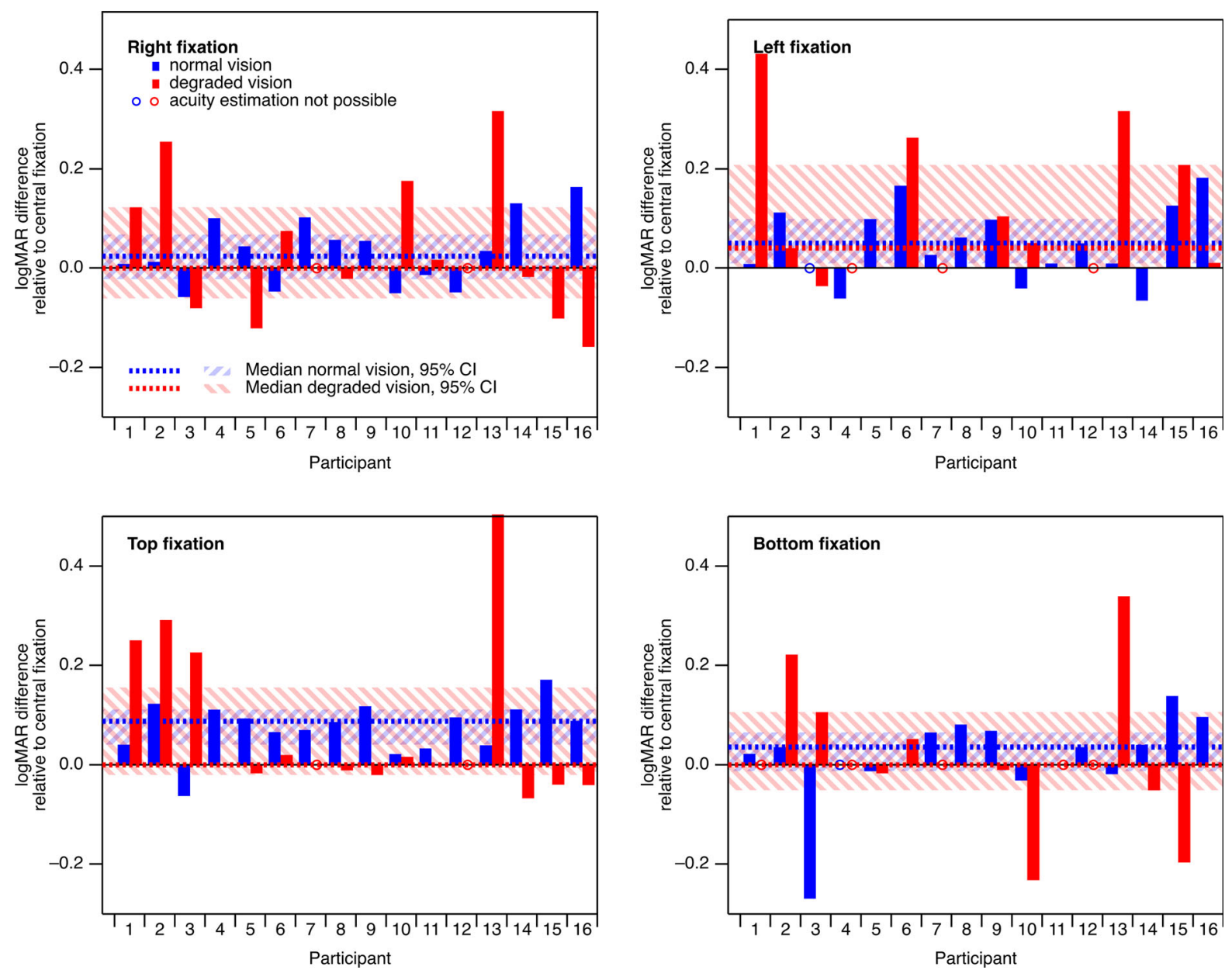

Fig. 3 Effect of eccentric fixation on acuity estimate in individual participants. In both normal vision (blue) and degraded vision (red), the median effects (dotted lines) were quite small and, with the exception of top fixation with normal vision, not significant

fixation, partly depending on the individual cortical structure, selecting only the $\mathrm{Oz}-\mathrm{O} 1$ or $\mathrm{Oz}-\mathrm{O} 2$ bipolar derivations from the Laplacian arrangement may yield further improvement [18].

The present findings are not unexpected, of course. The central visual field has a disproportionally large contribution to the VEP response [19]. In addition, especially with undegraded vision, near-threshold stimuli can only be resolved in the central visual field where acuity is highest. Thus, when the edge of the stimulus is fixated, the effective amount of neuronal stimulation near threshold can be expected to be halved or even further reduced. However, the effect on the VEP might be quite different, as evident from the fact that the responses may even increase with top fixation (Fig. 1). This is because the folding of the visual cortex, in particular at the calcarine sulcus, has a strong effect on the amount of activation that is picked up by an electrode and may result in a polarity inversion for some VEP components when stimulation is switched between the upper and lower visual field $[18,20]$. A visual inspection of the time course data across participants suggests a large variability of the VEP traces in this respect. In some participants, there was a polarity inversion between top and bottom fixation, which was absent in other participants. In some cases, the curve shapes differed in a way that cannot simply be described in terms of polarity or the effect depended on the check size. These differences may partly explain interindividual variability in the amount of amplitude change with eccentric fixation, particularly in the case of top and bottom fixation. 
While a general amplitude change should not have a sizable effect on the threshold of the VEP tuning curve, a dependence on check size could affect the outcome.

Given the stimulus dimensions in the present study, fixating the edge of the stimulus area results in a deviation from central fixation of about $7.5^{\circ}$ vertically or $9.5^{\circ}$ horizontally. This amount is large enough to be spotted by an experienced technician. It seems safe to assume that any deviation that is smaller and goes unnoticed will have less impact on the results than found in the present study. Thus, incorrect fixation will have little effect on acuity estimates. The present findings may also facilitate the interpretation of test outcomes in patients with eccentric fixation due to central visual field loss, for instance when using a preferred retinal locus [21].

In summary, the present data suggest that VEPbased acuity estimates are relatively insensitive to deviations from central fixation.

Acknowledgements We are grateful to Isabell Strübin and Verena Gauggel for their support, and we thank all participants for contributing to this study.

Funding Open Access funding enabled and organized by Projekt DEAL. This research was supported by the Bundesinstitut für Sportwissenschaft based on a decision of the Deutscher Bundestag (ZMVI4-070403/19-20).

Data Availability Data are available from the corresponding author upon reasonable request.

\section{Compliance with ethical standards}

Conflict of interest The authors declare that they have no conflict of interests.

Informed consent Informed consent was obtained from all individual participants included in the study.

Ethical approval All procedures performed in studies involving human participants were in accordance with the ethical standards of the institutional review board of the University of Freiburg.

Statement of human rights All procedures performed in studies involving human participants were in accordance with the ethical standards of the institutional review board of the University of Freiburg and followed the tenets of the Declaration of Helsinki.

Statement on the welfare of animals This article does not contain any studies with animals performed by any of the authors.
Open Access This article is licensed under a Creative Commons Attribution 4.0 International License, which permits use, sharing, adaptation, distribution and reproduction in any medium or format, as long as you give appropriate credit to the original author(s) and the source, provide a link to the Creative Commons licence, and indicate if changes were made. The images or other third party material in this article are included in the article's Creative Commons licence, unless indicated otherwise in a credit line to the material. If material is not included in the article's Creative Commons licence and your intended use is not permitted by statutory regulation or exceeds the permitted use, you will need to obtain permission directly from the copyright holder. To view a copy of this licence, visit http://creativecommons.org/licenses/by/4.0/.

\section{References}

1. Hamilton R, Bach M, Heinrich SP, et al (in press) VEP estimation of visual acuity: a systematic review. Doc Ophthalmol. https://doi.org/https://doi.org/10.1007/s10633020-09770-3

2. Strasser T, Nasser F, Langrová H et al (2019) Objective assessment of visual acuity: a refined model for analyzing the sweep VEP. Doc Ophthalmol 138:97-116. https://doi. org/10.1007/s10633-019-09672-z

3. Bach M, Heinrich SP (2019) Acuity VEP: Improved with machine learning. Doc Ophthalmol 139:113-122. https:// doi.org/10.1007/s10633-019-09701-x

4. Ridder WH, Waite BS, Melton TF (2014) Comparing Enfant and PowerDiva sweep visual evoked potential (sVEP) acuity estimates. Doc Ophthalmol 129:105-114. https://doi.org/10.1007/s10633-014-9457-7

5. Wenner Y, Heinrich SP, Beisse C et al (2014) Visual evoked potential-based acuity assessment: overestimation in amblyopia. Doc Ophthalmol 128:191-200. https://doi.org/ 10.1007/s10633-014-9432-3

6. Heinrich SP, Bock CM, Bach M (2016) Imitating the effect of amblyopia on VEP-based acuity estimates. Doc Ophthalmol 133:183-187. https://doi.org/10.1007/s10633-0169565-7

7. Ravensbergen HJCR, Mann DL, Kamper SJ (2016) Expert consensus statement to guide the evidence-based classification of Paralympic athletes with vision impairment: a Delphi study. Br J Sports Med 50:386-391. https://doi.org/ 10.1136/bjsports-2015-095434

8. Bach M (1996) The Freiburg Visual Acuity Test-automatic measurement of visual acuity. Optom Vis Sci 73:49-53

9. Odom JV, Bach M, Brigell M et al (2016) ISCEV standard for clinical visual evoked potentials: (2016 update). Doc Ophthalmol 133:1-9. https://doi.org/10.1007/s10633-0169553-y

10. Hamilton R, Bach M, Heinrich SP et al (in press) ISCEV extended protocol for VEP methods of estimation of visual acuity. Doc Ophthalmol. https://doi.org/10.1007/s10633020-09780-1

11. Bach M, Maurer JP, Wolf ME (2008) Visual evoked potential-based acuity assessment in normal vision, artificially degraded vision, and in patients. Br J Ophthalmol 92:396-403. https://doi.org/10.1136/bjo.2007.130245 
12. Bach M, Meigen T (1999) Do's and don'ts in Fourier analysis of steady-state potentials. Doc Ophthalmol 99:69-82. https://doi.org/10.1023/A:1002648202420

13. Meigen T, Bach M (1999) On the statistical significance of electrophysiological steady-state responses. Doc Ophthalmol 98:207-232. https://doi.org/10.1023/A:1002097208 337

14. Good PI (2006) Resampling Methods: A Practical Guide to Data Analysis, 3rd edn. Birkhäuser, Boston

15. Clayton MS, Yeung N, Cohen Kadosh R (2018) The many characters of visual alpha oscillations. Eur J Neurosci 48:2498-2508. https://doi.org/10.1111/ejn.13747

16. International Council of Ophthalmology (1988) Visual acuity measurement standard. Ital J Ophthalmol II/I:1-15

17. Manahilov V, Riemslag FC, Spekreijse H (1992) The laplacian analysis of the pattern onset response in man. Electroencephalogr Clin Neurophysiol 82:220-224. https:// doi.org/10.1016/0013-4694(92)90171-d

18. Russo FD, Martínez A, Sereno MI et al (2002) Cortical sources of the early components of the visual evoked potential. Hum Brain Mapp 15:95-111. https://doi.org/10. 1002/hbm. 10010

19. Klistorner AI, Graham SL (2005) Effect of eccentricity on pattern-pulse multifocal VEP. Doc Ophthalmol 110:209-218. https://doi.org/10.1007/s10633-005-7309-1

20. Clark VP, Fan S, Hillyard SA (1994) Identification of early visual evoked potential generators by retinotopic and topographic analyses. Hum Brain Mapp 2:170-187. https:// doi.org/10.1002/hbm.460020306

21. Crossland MD, Culham LE, Kabanarou SA, Rubin GS (2005) Preferred retinal locus development in patients with macular disease. Ophthalmology 112:1579-1585. https:// doi.org/10.1016/j.ophtha.2005.03.027

Publisher's Note Springer Nature remains neutral with regard to jurisdictional claims in published maps and institutional affiliations. 